\begin{tabular}{|c|c|c|c|c|}
\hline NIVEL & FIGURAS DE PROTECCIÓN & \multicolumn{3}{|c|}{ TIPO DE PROTECCIÓN } \\
\hline 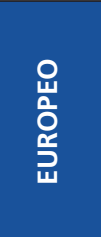 & $\begin{array}{l}\text { Lista Roja Europea de árboles } \\
\text { Hábitats de Interés Comunitario }\end{array}$ & \multicolumn{3}{|c|}{$\begin{array}{l}\text { Q. petraea y Q. robur: Menor preocupación } \\
\text { Incluidas en los Hábitats (no prioritarios): } \\
9230 \text { - "Robledales galaico-portugueses de Q. robur y Q. pyrenaica" } \\
9120 \text { - "Hayedos acidófilos atlánticos con sotobosque de llex y a veces de Taxus } \\
\text { (Quercionrobori-petraeae o llici-Fagenion)" }\end{array}$} \\
\hline $\begin{array}{l}\frac{1}{\nwarrow} \\
\frac{0}{z} \\
\text { 은 } \\
\frac{1}{z}\end{array}$ & $\begin{array}{l}\text { Atlas y Libro Rojo de la Flora Vascular } \\
\text { Amenazada de España } \\
\text { Listado de Especies Silvestres en Régimen } \\
\text { de Protección Especial y Catálogo Español } \\
\text { de Especies Amenazadas }\end{array}$ & \multicolumn{3}{|c|}{ Q. petraea y Q. robur no incluidas } \\
\hline \multirow{5}{*}{ 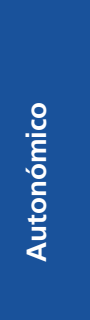 } & \multirow{4}{*}{$\begin{array}{l}\text { Catálogos Regionales de Especies de } \\
\text { Flora Amenazada }\end{array}$} & Comunidades & Q. petraea & Q. robur \\
\hline & & Castilla- La Mancha & Vulnerable & $\begin{array}{l}\text { Vulnerable (incluidos sus híbridos } \\
\text { con otros Quercus) }\end{array}$ \\
\hline & & Castilla y León & & No incluidas \\
\hline & & Madrid & Interés especial & Interés especial \\
\hline & Catálogos de Árboles Singulares & \multicolumn{3}{|c|}{ Comunidad de Madrid: 3 ejemplares de Q. petraea } \\
\hline
\end{tabular}

Tabla 1. Figuras de Protección de Quercus robur y Q. petraea en diferentes niveles administrativos

MAR GÉNOVA, ALICIA LÓPEZ, ELENA PAJARÓN, RUBÉN BERNAL y JOAQUÍN CASTELO Universidad Politécnica de Madrid, Depto. Sistemas y Recursos Naturales. C/ Ramiro de Maeztu S.n. 28040 Madrid.

\title{
Reproducción de Lllmus glabra Huds. en la región de procedencia 20: Sierra de Guadarrama-Ayllón Do: 10.15366/(20202024002
}

\begin{abstract}
The conservation status of Ulmus glabra Huds. in the Spanish central mountain range is endangered. Several studies report that the species is close to extinction in this area.

The aim of this project is to study the ex-situ reproduction of Ulmus glabra in nurseries in order to make available the plant material necessary to address conservation plans through the following partial objectives:

1.- Development of a protocol for the production of cloned specimens through vegetative reproduction by cutting, establishing the techniques that offer the best rooting results.

2.- Analysis of the capacity of sexual reproduction and the viability of the seeds.
\end{abstract}

Samples of 49 trees were taken from 9 different populations and the influence of 3 factors on rooting success was studied, concluding that only the use of indolbutyric acid was statistically different than the rest.

Wych elm viable seeds are very rare, but contrary to previous reports they are easily conserved and even maintain their germination capacity for at least one year in outdoor nursery growing conditions (heat, cold and high humidity).

Palabras clave: Olmo de montaña, Sistema Central, España, conservación, reproducción vegetativa, semilla.

\section{Introducción}

El estado de conservación de Ulmus glabra (olmo de montaña) en el Sistema Central es preocupante. Martín del Puerto (2017) analizó su situación en esta Cordillera con la metodología UICN (2012), y obtuvo que el taxón se encuentra ante un elevado riesgo para su supervivencia a nivel regional (se le podría catalogar como En Peligro).

La fragmentación severa de sus poblaciones, el escaso reclutamiento y la elevada mortalidad, especialmente por grafiosis, son sus principales factores de amenaza. Cuando el grado de deterioro de la población es avanzado y existe el peligro de desaparición de algunos genotipos, además de los trabajos in situ, es necesario adoptar medidas de conservación ex situ (Fernández et al., 2000) como la colecta de material vegetal y su mantenimiento en vivero en forma de colección, creando así una reserva para su investigación y cultivo con fines de traslocación in situ.

El área de este estudio es la Región de Procedencia 20. Sierra de Guadarrama-Ayllón (Alía et al., 2009), donde Ulmus glabra cuenta solo con unos 300 ejemplares reunidos en 20 pobla- 


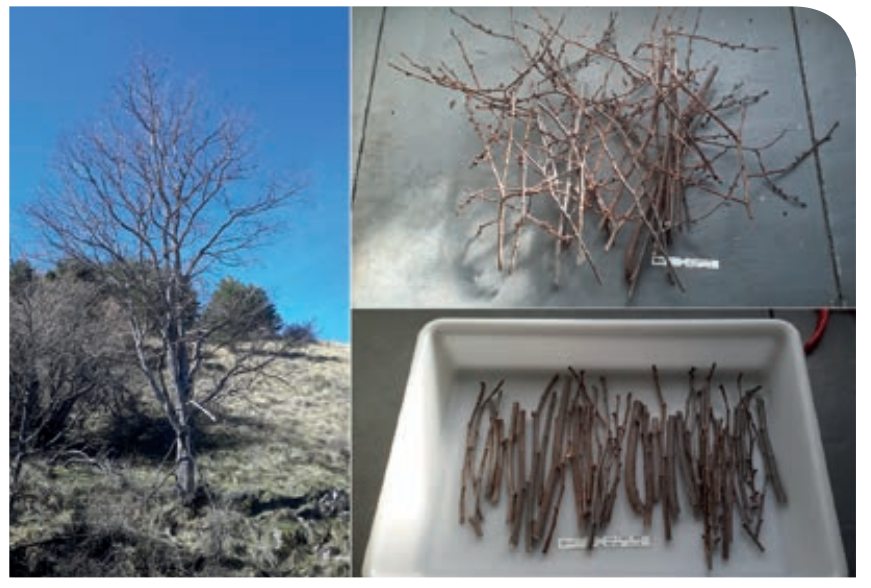

Figura 1. Ejemplar $n^{\circ} 5$ de La Morcuera el de mayor tamaño de Madrid: $h: 20$ m. d:60 cm. Izda. en campo. Arriba: estaquillas tomadas. Abajo: estaquillas preparadas. (Foto I. Colmenero).

ciones, casi la mitad de éstas con un solo individuo (Tabla 1). Ante esta situación se están empezando a plantear medidas de conservación de la especie en el Parque Nacional de la Sierra de Guadarrama. En este trabajo se ha estudiado su reproducción en vivero con el objetivo de poner a disposición de los gestores el material vegetal necesario para afrontar los planes de conservación. Todo ello mediante los siguientes objetivos parciales:

1.- Elaboración de un procedimiento de producción de ejemplares clónicos mediante la reproducción vegetativa por estaquillado, estableciendo las técnicas que ofrecen mejores resultados de enraizamiento.

2.- Análisis de la capacidad de reproducción sexual y de la viabilidad de las semillas.

\section{Material y métodos}

Se ha colectado material de 49 ejemplares de un total de 332 que viven en el área de estudio: en 3 poblaciones de Guadalajara, 1 de Segovia y 5 de Madrid (Tabla 1). Para analizar su capacidad de regeneración vegetativa y evaluar las técnicas que ofrecen mejores resultados de enraizamiento se han preparado 784 estaquillas (Figura 1), que se han puesto a enraizar en diferentes condiciones que determinan el éxito del enraizamiento: i) El tipo de sustrato (perlita sola o mezcla de perlita y sustrato a base de turba en proporción 2/1), ii) Heridas adicionales (con corte en la base o sin corte) y iii) Diferentes concentraciones de hormona de enraizamiento ácido indolbutírico $\operatorname{AIB}$ (0, 3.000, 6.000 y 9.000 ppm).

La combinación de las 8 variables de los 3 factores supone un total de 16 tratamientos: Las 2 variables del tipo de sustrato muestran la influencia de la porosidad y la retención de agua; las 2 variables de la inducción del enraizamiento mediante heridas, la relevancia de las condiciones traumáticas en la formación de primordios radiculares; y las 4 variables de concentración de hormona de enraizamiento, muestran primero si su aplicación es o no útil, y en caso positivo, su concentración más efectiva. Las 16 combinaciones de variables de los 3 factores se aplicaron a las estaquillas de los 49 ejemplares muestreados. Todas se colocaron en una mesa de enraizamiento con calor de fondo y riego por nebulización (Figura 2).

Para analizar el éxito de la reproducción sexual se ha estudiado la viabilidad, capacidad de germinación y conservación de las semillas. A lo largo de varios años se ha observado una baja fructificación y viabilidad de las semillas de estas poblaciones. Para este trabajo se estudió un lote de 83 gr /
8.057 semillas de 9 árboles distintos recolectadas en junio. La mitad se sembró recién recogida y la otra mitad se secaron al aire y se almacenaron en nevera a $4^{\circ} \mathrm{C}$ y se sembraron en la primavera siguiente (marzo).

\section{Resultados}

De las 784 estaquillas se produjo la brotación foliar de 472, el 60,2\%, aunque sólo han resultado exitosas, al emitir raíces (tabla 2), 95 estaquillas, un 12,11\% (Figura 2).

El análisis de la varianza de diseño factorial completo de los 3 factores estudiados y sus combinaciones no ofrecen datos estadísticamente significativos en los resultados de enraizamiento con el tipo de sustrato ni con la inducción por heridas, pero sí con la aplicación de hormona de enraizamiento, encontrándose una clara diferencia entre aplicarla o no. Respecto a la concentración, la más eficaz ha sido la de 3.000 ppm, aunque en tan poca diferencia con las de 6.000 y 9.000 ppm que no fue significativa.

La escasa fructificación y la abundancia de semillas vanas observadas en la población de La Morcuera coinciden con otros estudios realizados sobre su potencial reproductivo en el Sistema Central (Maqueda, 2006; Arche de Miguel, 2010).

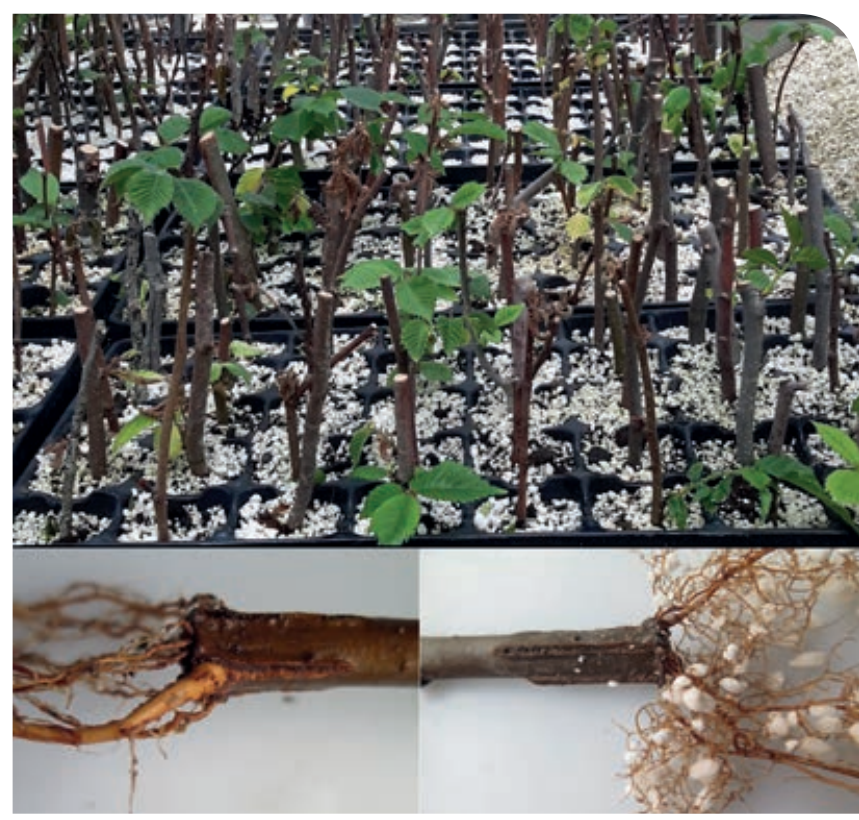

Figura 2. Estaquillas en mayo con brotes vivos y muertos y enraizados en septiembre. (Foto I. Colmenero).

Los análisis de la viabilidad de las semillas colectadas para de este estudio muestran que la proporción de vanas es muy alta, como media en torno al 90\%, llegando en algunos árboles al $99 \%$.

Tanto la siembra de junio como la de marzo del año siguiente permanecieron en vivero dos años y en ambos casos pudimos constatar que la semilla no pierde su viabilidad, aunque no germine el primer año, pues hubo un nuevo ciclo de germinaciones con ambos lotes de semilla después de pasar un año sembradas en condiciones de vivero (calor, frío y humedad) (tabla 3, Figura 3).

\section{Discusión}

Se ha conseguido poner a punto una técnica de reproducción clonal que hasta la fecha no existía. Esto es un hecho muy relevante para la conservación de las poblaciones relícticas meridionales de la especie ante su alarmante situación. 


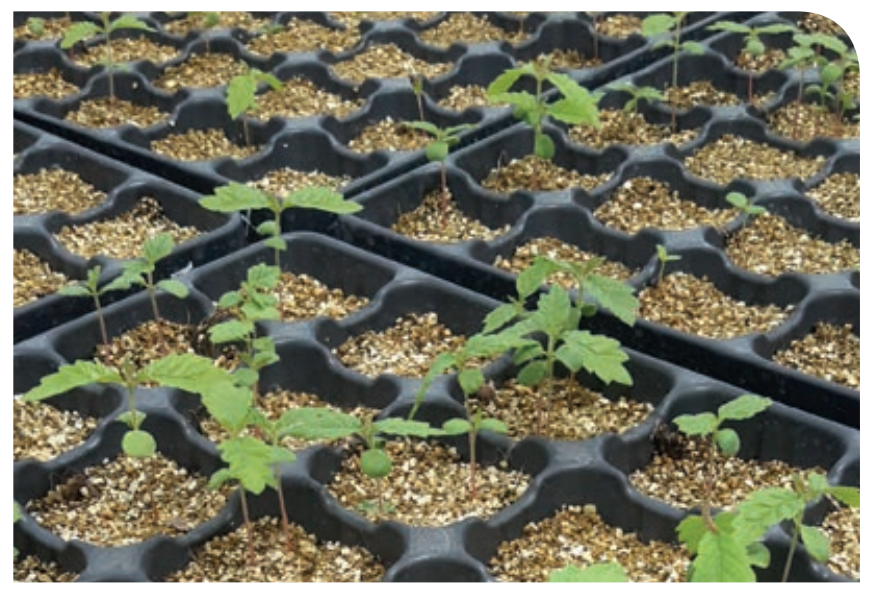

Figura 3. Germinación a 35 días de la siembra (Foto I. Colmenero).

Se han obtenido clones de 34 olmos (el $10 \%$ de la población estudiada) y 275 ejemplares procedentes de semilla. Este es un material muy valioso que ha permitido abordar la siguiente fase del programa de conservación del olmo de montaña en el Parque Nacional de la Sierra de Guadarrama, con la plantación in situ en la población de la Morcuera, con ejemplares de la procedencia genética adecuada.

Hasta la actualidad se pensaba que las semillas de esta especie pierden viabilidad después de 6 meses (Crocker, 1938), y que ésta se podía mantener mediante almacenamiento frío, hermético y seco (Grime et al., 1981). Nuestros resultados muestran que en determinadas condiciones las semillas si conservan su capacidad de germinación más allá de un año. Esto abre una nueva línea de estudio para identificar la causa de los problemas de la regeneración natural mediante el banco de semillas del suelo en las poblaciones silvestres, aparte de los ya conocidos de la fructificación irregular y el alto porcentaje de semillas vanas.

\section{Conclusiones}

- Es factible obtener ejemplares clónicos de Ulmus glabra mediante estaquillado.

- De las variables evaluadas para inducir el enraizamiento, el mejor tratamiento con AIB es 3.000 ppm. El tipo de sustrato y las lesiones adicionales no muestran resultados significativos.

- Las semillas de Ulmus glabra, en contra de lo que se pensaba, conservan la capacidad germinativa al menos un año incluso en condiciones de cultivo.

\section{Agradecimientos}

A los trabajadores de los organismos que han colaborado en los trabajos de campo: Parque Nacional de la Sierra de Guadarrama, IMIDRA, Parque Natural de la Sierra Norte de Guadalajara, Servicio Territorial de Medio Ambiente en Segovia y sus Agentes Medioambientales.

\begin{tabular}{|c|c|c|c|c|c|}
\hline POBLACIÓN/MUNICIPIO & ALTITUD (m) & $\mathrm{N}^{\circ}$ INDIVIDUOS & $\begin{array}{l}\text { INDIVIDUOS } \\
\text { MUESTREADOS }\end{array}$ & $\begin{array}{l}\text { INDIVIDUOS } \\
\text { CLONADOS }\end{array}$ & $\begin{array}{l}\text { ESTAQUILLAS } \\
\text { ENRAIZADAS }\end{array}$ \\
\hline La Calera / Cantalojas (GU) & 1.393 & 1 & & & \\
\hline Arroyo de la Zarza / Cantalojas (GU) & 1.557 & 194 & 13 & 8 & $7,7 \%$ \\
\hline Garganta Luenga / Cantalojas (GU) & 1.650 & 13 & & & \\
\hline Peñalba de la Sa / El Cardoso de la Sa (GU) & 1.593 & 1 & 1 & 1 & $14,0 \%$ \\
\hline Bocígano / El Cardoso de la Sierra (GU) & 1.314 & 8 & 1 & 1 & $1,9 \%$ \\
\hline Retiendas (GU) & 850 & 1 & & & \\
\hline Valverde de los Arroyos (GU) & 1.250 & 9 & & & \\
\hline Becerril/ Riaza (SG) & 1.556 & 27 & & & \\
\hline Navafría (SG) & 1.512 & 14 & 7 & 6 & $10,9 \%$ \\
\hline Arcones (SG) & 1.600 & 1 & & & \\
\hline Torre Val de San Pedro (SG) & 1.650 & 1 & & & \\
\hline Santo Tomé del Puerto (SG) & 1.500 & 1 & & & \\
\hline Montejo de la Sierra (M) & 1.463 & 9 & 4 & 1 & $3,0 \%$ \\
\hline Puebla de la Sierra (M) & 1.120 & 1 & & & \\
\hline Morcuera / Rascafría (M) & 1.451 & 37 & 14 & 8 & $4,6 \%$ \\
\hline Calderuelas / Rascafría (M) & 1.670 & 1 & 1 & 1 & $61,7 \%$ \\
\hline Arroyo Valhondillo / Rascafría (M) & 1.860 & 2 & & & \\
\hline Bustarviejo (M) & 1.570 & 1 & & & \\
\hline Manzanares el Real (M) & 1.598 & 4 & 4 & 4 & $33,7 \%$ \\
\hline Cercedilla (M) & 1.260 & 6 & 4 & 4 & $27,0 \%$ \\
\hline
\end{tabular}

Tabla 1. Poblaciones de Ulmus glabra incluidas dentro de la Región de Procedencia 20 Sierra de Guadarrama-Ayllón

\begin{tabular}{|c|c|c|c|c|}
\hline \multicolumn{5}{|c|}{ ESTAQUILLAS ENRAIZADAS EN CADA BANDEJA } \\
\hline & \multicolumn{2}{|c|}{ PERLITA } & \multicolumn{2}{|c|}{ PERLITATTURBA } \\
\hline & SIN CORTE & CON CORTE & SIN CORTE & CON CORTE \\
\hline AlB 0 ppm & 0 & 0 & 1 & 1 \\
\hline AIB 3.000 ppm & 9 & 8 & 9 & 9 \\
\hline AIB 6.000 ppm & 6 & 9 & 6 & 8 \\
\hline AlB 9.000 ppm & 8 & 8 & 4 & 9 \\
\hline
\end{tabular}




\begin{tabular}{|c|c|c|c|}
\hline & $\begin{array}{c}\text { Germinaciones } \\
\text { en la } 1^{\mathrm{a}} \text { primavera }\end{array}$ & $\begin{array}{c}\text { Germinaciones } \\
\text { en la } 2^{\mathrm{a}} \text { primavera }\end{array}$ & TOTAL \\
\hline $\begin{array}{l}\text { Semillas sembradas } \\
\text { recién recolectadas }\end{array}$ & $41(6 \%)$ & $24(4 \%)$ & $65(10 \%)$ \\
\hline $\begin{array}{r}\text { Semillas sembradas } \\
\text { después de un año conservadas en frío }\end{array}$ & $184(30 \%)$ & $26(4 \%)$ & $210(34 \%)$ \\
\hline
\end{tabular}

Tabla 3. Periodo de germinación de cada lote (porcentaje respecto a las semillas viables sembradas en cada periodo).

\section{Bibliografía}

- Alía, R., García del Barrio, J. M., Iglesias, S, Mancha, J. A., de Miguel, J., Nicolás, J.L., Pérez, F., Sánchez, D. (2009). Regiones de Procedencia de especies forestales en España. Ministerio de Medio Ambiente y Medio Rural y Marino.

- Arche de Miguel, J. (2010). Censo, corología y estudio dendrométrico de Ulmus glabra Huds. en el Sistema Central español. Estado de Conservación. Proyecto Fin de Carrera. E.T.S.I. Montes, UPM. Madrid

- Crocker, W. (1938). Life-span of seeds. Botanical Review, 4: 235-274.
- Fernández López, J. Díaz Vázquez, R. Cogolludo Agustín, M. A. Pereira Lorenzo, S. (2000). Conservación de Recursos Genéticos de las frondosas nobles en España. Invest. Agr.: Sist. Recur. For.: Fuera De Serie 2: 71-93

- Grime J.P. Mason, G Curtis, A.A. Rodman, J Band, S.R., Mowforth, M.A.G., Neal, A.M. and Shaw, S. (1981). A comparative study of germination characteristics in a local flora. Journal of Ecology, 69:1017-1059.

- Maqueda, A. (2006). Estudio de la población de olmo de montaña (Ulmus glabra Huds) en el Valle de Iruelas (Ávila). Proyecto Fin de Carrera. E.T.S.I Montes, UPM. Madrid.

- Martín del Puerto, M. (2017) Caracterización de las poblaciones de Ulmus glabra Hudson en el Sistema Central. Elaboración de propuestas para su Gestión Agronómica, Agroalimentaria y de Biosistemas. Agronomica, Agroalimentaria y de Biosistemas. UPM. Madrid.

- UICN. (2012). Categorías y Criterios de la Lista Roja de la UICN: Versión 3.1. Segunda edición. Gland, Suiza y Cambridge, Reino Unido: UICN. vi + 34pp.

ISIDORO COLMENERO MARTÍN1', FELIPE MARTÍNEZ GARCÍA²

1. Instituto Madrileño de Investigación y Desarrollo Rural, Agrario y Alimentario (IMIDRA) C/ Leganitos, 47 - 28013 Madrid (isidoro.colmenero@madrid.org) 2. Departamento de Sistemas y Recursos Naturales. Escuela Técnica Superior de Ingeniería de Montes, Forestal y del Medio Natural. Universidad Politécnica de Madrid. Paseo de las Moreras s/n, 28040 Madrid. (felipe.martinez@upm.es)

\section{Máximo riesgo}

\section{Lotus gomerythus A. Portero, J. Martín- [arbajal \& R. Mesa, historia del descubrimiento de una nueva especie para La Gomera}

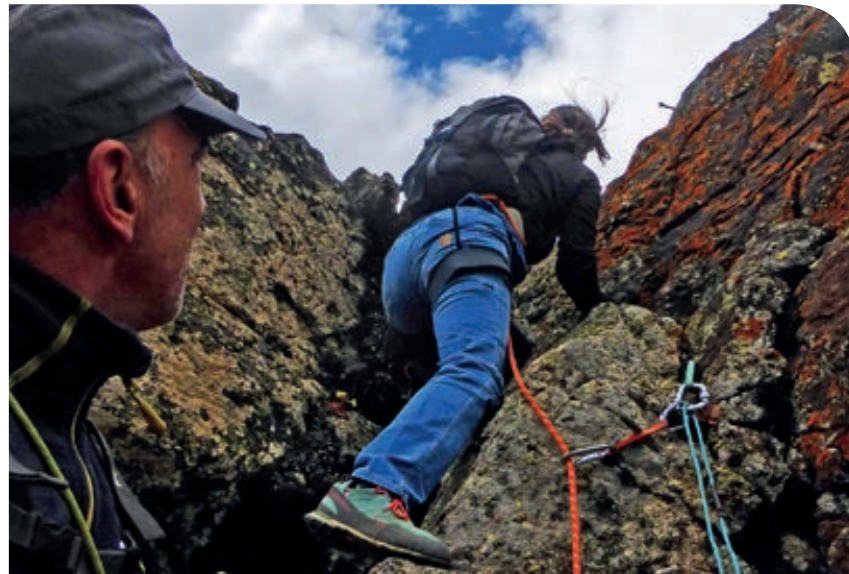

Figura 1. Ascensión al roque del Sombrero utilizando técnicas de escalada (Foto: J. Martín-Carbajal).

Fue una acción de control de la especie exótica invasora Cenchrus orientalis (Rich.) Morrone (rabogato) la que nos llevó a la isla de La Gomera aquel fin de semana de febrero de 2016. La Asociación Ambiental Matorrisco, daba sus primeros pasos en la isla con un objetivo similar al de la Asociación Abeque y solicitaba la colaboración y experiencia acumulada por nuestro grupo, tanto en la planificación de las acciones como en el manejo de esta planta invasora. Abeque nació en Tenerife, en el año 2011, fruto de la inquietud de un grupo de personas que sentíamos la necesidad de comprometernos activamente en la conservación del patrimonio natural de las Islas Canarias. Decidimos centrar nuestros esfuerzos en el macizo de Teno, uno de los espacios naturales más emblemáticos de Canarias con un alto valor botánico y faunístico situado al noroeste de Tenerife. Nuestro principal objetivo era la lucha contra las especies exóticas invasoras y las acciones van dirigidas, sobre todo, al control del rabogato que, en aquel momento, dominaba varios enclaves de dicho territorio. A lo largo de estos años, gracias a la resistencia y perseverancia de un fiel voluntariado, en coordinación con las administraciones locales, Ayuntamiento de Buenavista del Norte y Oficinas de Gestión del Parque Rural de Teno, hemos logrado resultados muy exitosos en la ardua tarea de control y eliminación de esta especie invasora que exige constancia y un riguroso protocolo (Portero et al., 2018).

Acabado nuestro compromiso con ambas asociaciones durante aquel fin de semana, nos dispusimos a hacer una excursión en la vertiente sureste de la isla de La Gomera, que 